\title{
Double-outlet right ventricle is not hypoplastic left heart syndrome
}

To the Editor - We write to express our concerns that the report 'The complex genetics of hypoplastic left heart syndrome' by Liu et al. ${ }^{1}$ is misleading, and the data presented in the figures and in the Mouse Genome Informatics repository do not convincingly demonstrate hypoplastic left heart syndrome (HLHS).

An international nomenclature committee has specifically defined this particular lesion ${ }^{2}$. This definition is now contained within the Eleventh Revision of the International Classification of Diseases (ICD-11) $)^{3}$. It is the combination of a small non-apex-forming left ventricle, hypoplasia of the ascending aorta, and a combination of aortic and mitral valve stenosis or atresia. The definition requires the presence of concordant ventriculoarterial connections. Hence, the aorta must arise from the left ventricle, and the pulmonary trunk must arise from the right ventricle. The definition specifically excludes diagnoses such as double-outlet right ventricle (DORV), transposition of the great arteries (TGA), common arterial trunk (CAT), and atrioventricular septal defects (AVSD), all of which on occasion can be associated with a small left ventricle.
Liu et al. refer to the CRISPR-Cas9 mutants as the "double-outlet RV variant of HLHS," which is a contradiction ${ }^{4}$. The appearances of these mice are similar to that of a well-recognized but rare form of DORV with a restrictive or closed interventricular communication $^{5}$. Double outlet from the right ventricle is the normal situation in the early embryo, and a lack of transfer of the aorta into the left ventricle redefines the arrangement as a malformation ${ }^{6,7}$. In HLHS, because there are concordant ventriculoarterial connections by definition, transfer of the aortic root must have taken place $^{8}$. TGA, CAT, and AVSD all result from different developmental processes?

It follows that the suggestion that digenic inheritance is likely to be responsible for HLHS is without foundation, because none of the mice described have HLHS. The human genomic data within the paper do not independently support the conclusion of digenic inheritance. The atypical protocadherin implicated in the patient is not homologous to that described for the mouse model, nor is any clinical information provided.

Our overall tenet is that it is inappropriate to reclassify mutants with DORV as HLHS and that, in doing so, this report may risk misleading clinicians, families affected by HLHS, and researchers seeking to understand specific etiologies underlying HLHS.

\section{Bill Chaudhry*, Deborah Henderson* and Robert Anderson* \\ Cardiovascular Research Centre, Institute of Genetic Medicine, Newcastle University, Newcastle upon Tyne, UK. \\ *e-mail:bill.chaudhry@ncl.ac.uk; deborah. \\ henderson@ncl.ac.uk;sejjran@ucl.ac.uk}

Published online: 7 January 2019

https://doi.org/10.1038/s41588-018-0324-4

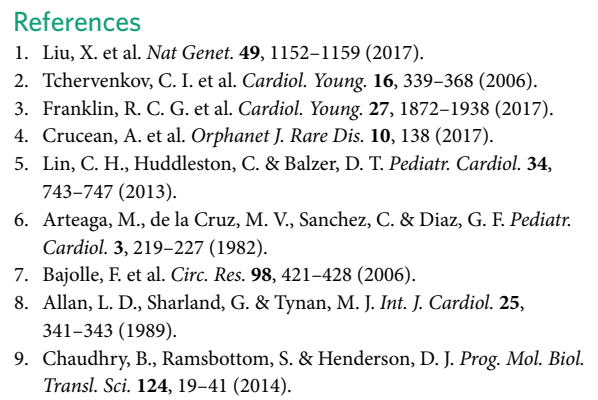

\section{Reply to 'Double-outlet right ventricle is not hypoplastic left heart syndrome'}

Lo et al. reply - Our documentation of the presence of hypoplastic left heart syndrome (HLHS) in mutant mice was based on three levels of analyses ${ }^{1}$. First, fetal echocardiography was performed, which yielded evidence of HLHS on the basis of the combination of 2D and color-flow imaging and hemodynamic assessments (Fig. 1a,b and Supplementary Videos 1 and 2 in ref. ${ }^{1}$ ). Second, necropsy was performed, which provided direct visualization of the left ventricle (LV) and aorta (Fig. 1c and Supplementary Video 3 in ref. $\left.{ }^{1}\right)$. Third, confocal histopathology was carried out with episcopic confocal microscopy for 3D reconstructions to assess intracardiac anatomy (valves and inflow/outflow connections) (Fig. 1d and Supplementary Video 4 in ref. ${ }^{1}$ ). Hence, the diagnosis of HLHS in each mutant was based on a thorough threeprong analysis: functional analysis with fetal echocardiography, and anatomical analysis with necropsy and $3 \mathrm{D}$ reconstructions.

We have no disagreement with the definition of HLHS described by Chaudhry et al. ${ }^{2}$. Indeed, the mutants from the Ohia mouse line recovered from our screen precisely fit this classic definition of HLHS. Thus, in the data in Table 1 of ref. ${ }^{1}, 23$ (82\%) of the 28 HLHS mutants recovered from the Ohia-mutant line had classic HLHS as defined above. We also duly noted (footnote 'a' in Table 1) that 5 of the 28 mutants had double-outlet right ventricle (DORV) in conjunction with hypoplastic LV. We further observed classic HLHS and also DORV with hypoplastic LV in the Sap130; Pcdha9 CRISPR-mutant mice, thus confirming that both phenotypes are heritable and are elicited by the Sap130; Pcdha9 mutations. Consequentially, these genetic findings challenge the definition of HLHS based solely on cardiac morphology.

The heterogeneity of cardiac phenotypes in genetic studies of congenital heart disease (CHD) has been challenging. We note that the phenotypic variation observed in Ohia mutants is on par with the spectrum of 